\title{
WI-ALERT : A WIRELESS SENSOR NETWORK BASED INTRUSION ALERT PROTOTYPE FOR HEC
}

\author{
Ruwini Edirisinghe ${ }^{1}$, Dileeka Dias ${ }^{2}$, Rakhitha Chandrasekara ${ }^{3}$, Lanka Wijesinghe ${ }^{3}$, \\ Prasanga Siriwardena ${ }^{3}$ and Prasad Kumara Sampath ${ }^{3}$ \\ ${ }^{1}$ RMIT University, Melbourne, Australia \\ ${ }^{2}$ University of Moratuwa, Sri Lanka \\ ${ }^{3}$ Dialog-University of Moratuwa Mobile Communications Research Laboratory
}

\begin{abstract}
A wired fence based intrusion detection and alerting mechanism for boundaries separating wildlife habitats and human settlements was implemented, particularly as a solution to the Human-Elephant Conflict (HEC). The objective of the research reported in this paper is to propose and verify an alternative technique for this wired fence based alerting mechanism to overcome its limitations and to improve its effectiveness.

This article presents a comprehensive study of alternative solutions with deliberate consideration of the practical constraints. Wi-Alert is a wireless sensor network based intrusion detection system proposed as the best alternative solution. This article reports the outcomes of the first two phases of ongoing developments of Wi-Alert.

The first phase of experiments was conducted to investigate the multi-path effect reduction techniques at one site. In the next phase, experiments were conducted to verify the ability to detect elephants. The results obtained via the candidate techniques are compared. Both experiments confirm the feasibility of the prototype as a non-invasive method to detect elephants.
\end{abstract}

\section{KEYWORDS}

Human-Elephant Conflict, sensor network, LOS obstruction, Intrusion Detection, multi path effect.

\section{INTRODUCTION}

The conflict between humans and elephants have been reported as a serious socio-economic problem in various regions in Africa [1-4] and Asia [5]. The Human-Elephant Conflict (HEC) has intensified in the recent past causing severe negative impacts on each group; humans and elephants.

A study[6] provided a broader view of the socio-political and ecological dimensions of HEC, in particular the causes for intensifying the conflict. The authors argue that 'change of land use' due to various activities including spread of agriculture into previously unoccupied wildlife habitat, 'changes of elephant behaviour and socio-ecology due to human intervention' and 'changes in social relationships in rural communities' are the social and physical conditions which exacerbate the conflict that has always existed between elephants and agriculturalists. 
Elephants coming in to contact with humans, impact negatively in various means such as by depredation of crops, damaging grain stores, water supplies, houses and other assets, injuring and death of humans and elephants.

Humans killed by elephants are reported in India [7,8] in Kenya [3] and in Uganda[6]. Also, as a result of HEC, humans kill elephants by poisoning or through other elephant control campaigns[7,9]. A study done in India reports that up to $20 \%$ of elephant deaths were caused directly by crop defence[5].

In Sri Lanka, HEC is the biggest environmental \& socio economic problem as shown in Figure 1(a) and Figure 1(b). In Sri Lanka, 70\% of the wild elephant populations live outside the wildlife sanctuaries, sharing the land with rural people and agriculturalists. As a result, 3 million people in 13 districts in Sri Lanka are affected by HEC. During the period from 1992 to 2008, total of 914 humans were killed by elephants and 2,337 elephants were killed by farmers and during the period from 2004 to 2007 a total of 3,103 homes were destroyed by elephants. Since 2008, 225 elephants are being killed annually on average in Sri Lanka. The damages caused by elephants to crops in the area have been estimated to cost Rs. 1,100 million (approx. US\$10 million) annually[10].

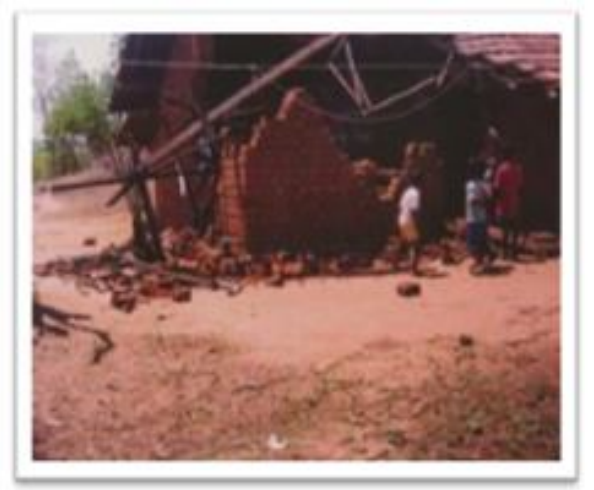

(a) Elephant attacks to a house in human settlement

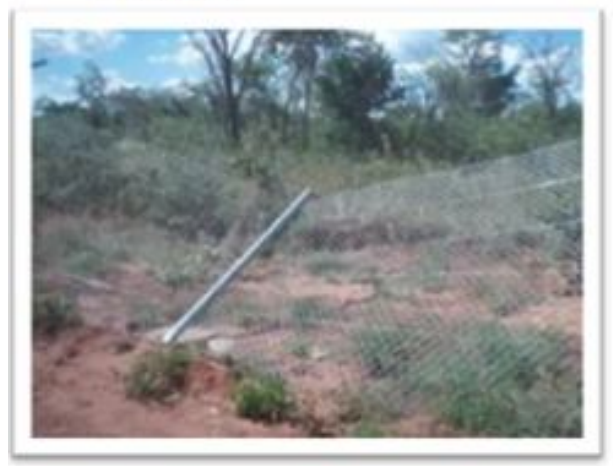

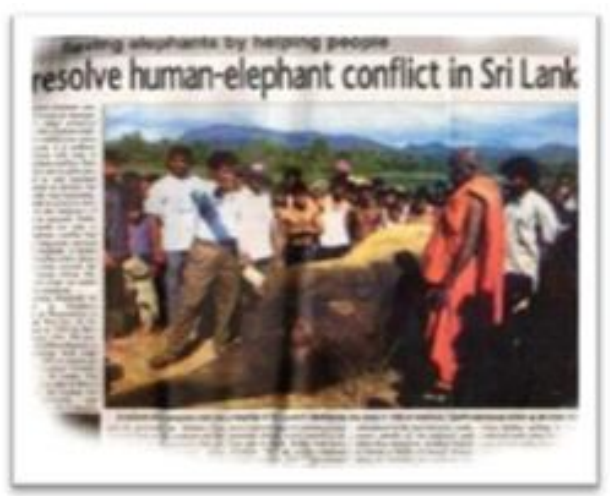

(b) $\mathrm{HEC}$ is a major environmental \& Socio Economic problem is Sri Lanka

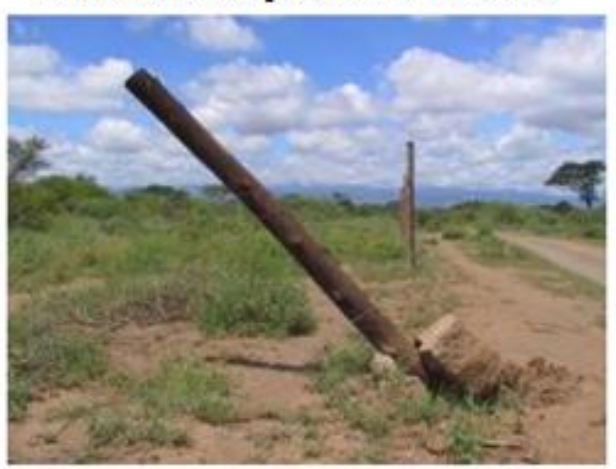

(C) Attacks to electric fences by Elephants

Figure 1. HEC in Sri Lanka

Providing effective solutions for human-elephant conflict is now one of the most significant challenges for the elephant management all over the world. For example, African Elephant 
Specialist Group (AfESG) which is a specialist group of the International Union for Conservation of Nature (IUCN) has assigned a task force called Human-Elephant Conflict Working Group (HECWG) to address the HEC.

Various technological solutions have been developed from time to time to overcome limitations of the basic techniques used by agriculturalists at the boundaries of human settlement. The electric fencing system used in Sri Lanka is frequently attacked by elephants, placing both humans and elephants at risks. eleAlert [10], the intrusion detection and alerting mechanism was implemented as a solution to this problem. However, due to several practical limitations of the eleAlert, the research team sought an alternative solution for its improvement.

The research reported in this paper is composed of two parts. Firstly, it investigates and reviews the possible alternative techniques considering related economic, social and technological constraints. Based on the above analysis this study proposes the best alternative solution. Secondly, the best alternative solution is designed to be implemented in three phases. This paper reports the first two phases of the project: feasibility study and prototype testing.

The remainder of the paper discusses various techniques reviewed and proposes Wi-Alert, which is a wireless sensor network based intrusion alert system. The paper also presents the prototype development and testing. The experiments conducted at two sites and the results are discussed. Finally, the paper presents the concluding remarks and the future work.

\section{BACKGROUND AND RELATED WORK}

The traditional approaches used by humans at the boundaries of wildlife habitats and human settlements to protect themselves from elephant attacks include lighting fires and fire crackers, making loud sounds and digging trenches to scare the elephants.

A reasonably technical approach commonly introduced to control the movement of elephants worldwide is electric fences. Over $1000 \mathrm{~km}$ has been erected with electric fences in Sri Lanka with over 300km being erected in 2009[10]. Fences continue to be erected with rapid development taking place in rural Sri Lanka at present. In electric fences elephants are deterred from forcing through the fence by an electric shock. The elephants tend to respect the fence and it acts as a psychological barrier. The electric fencing is costly to install (for example approximately $0.5 \mathrm{M}$ Sri Lankan Rs. per $\mathrm{Km}$ ) and difficult to maintain too. Also, the elephants become acclimatized and/or overcome the system by ingenious means (for example short-circuiting electrified fence with upturned tree trunks). Hence, the electric fences are vulnerable to attacks as shown in Figure 1(c). The electric fencing solution has no mechanism for immediate alerting of breaches, and locating faults is tiresome and difficult. As a result $90 \%$ of over $1000 \mathrm{~km}$ of existing fences in Sri Lanka are not functioning.

eleAlert was designed to overcome the limitations of the electric fencing solution, to provide a mechanism to detect intrusions in real time, to generate immediate alerts of intrusions and also to locate the intrusion. eleAlert was implemented and tested in elephant habitats demarcated by electric fences. After succeeding pilot trials and several versions of the system, it was fine tuned to improve sensing and communication mechanisms and the power efficiency. As a result the power efficiency was improved so that bridges can run for approximately 6 months, and the Remote Transmitting Unit (RTU which is the gateway between the system and the mobile network) can run for over 5 consecutive days without the solar power [10].

However, the current eleAlert system has some limitations in facilitating continuous monitoring of the elephant behaviour over time. The major challenge is the physical access to the system for restoration after an intrusion. Hence, an alternative technique is sought. 
The research team reviewed all possible alternative techniques considering the pros and cons of each, with deliberate consideration to the implementation challenges and/or limitations.

The alternative solutions to eleAlert are subject to technical restrictions that can be imposed by the harsh rural environment, as well as economic and social constraints imposed by the elephant behaviour and attitudes, perceptions as well as the behaviour of the human settlements.

We do not consider tagging elephants as an option for this study. Instead, the major concern is that the techniques should be non-invasive. The system should also consider the technical development challenges. Additional technical constraints associated with the system are as follows. It should be composed of minimal installation complexity in the site (rural area/forest). In addition, the on-site testing should not be complicated. False alarms of the system due to other intruders (for e.g. birds and other animals) should be handled. Moreover, the system should be reasonably energy efficient as the power supply is a significant parameter in real implementation. More importantly, easy maintenance particularly after the intrusion is a critical factor. Finally, because hundreds (in some areas, thousands) of kilometre length exists in the boundary of elephant habitat and human settlement, the system scalability is also crucial. The main economic aspect is the cost efficiency. Some social constraints also exist which are involved with the security of the system. Sufficient remedy should be implemented for reasonably expensive components of the system to protect them from hold-up. Table 1 summarises these intrusion detection techniques reviewed and they are further discussed below.

Table 1. Alternative Techniques

\begin{tabular}{|c|l|l|}
\hline Technique & \multicolumn{1}{|c|}{ Positives } & \multicolumn{1}{c|}{$\begin{array}{c}\text { Practical Limitations and } \\
\text { Challenges }\end{array}$} \\
\hline Light and Camera & Non-invasive, Economical & $\begin{array}{l}\text { System reliability, Complex } \\
\text { installation }\end{array}$ \\
\hline Ultrasound & $\begin{array}{l}\text { Non-invasive, Easy to } \\
\text { locate, Information rich data }\end{array}$ & $\begin{array}{l}\text { Advance rotating techniques needed, } \\
\text { limitations on distance }\end{array}$ \\
\hline $\begin{array}{c}\text { Infra sound and } \\
\text { Seismic }\end{array}$ & $\begin{array}{l}\text { Accurate locating, added } \\
\text { advantage of chasing } \\
\text { elephants back }\end{array}$ & $\begin{array}{l}\text { Hardware complexity and cost, power } \\
\text { issues, domain expertise }\end{array}$ \\
\hline $\begin{array}{c}\text { Wireless sensors } \\
\text { Flexible, easy installation, } \\
\text { easy maintenance }\end{array}$ & $\begin{array}{l}\text { Thorough testing needed for reliability } \\
\text { and robustness of final product, } \\
\text { configuration and positioning } \\
\text { challenges, power issues }\end{array}$ \\
\hline
\end{tabular}

\subsection{Light and Camera Option}

Kays et al.[11] used arrays of modern motion sensitive camera traps to monitor wild animals. Modern digital camera traps that record video, present new analytical opportunities, but also new data management challenges [11]. However, due to the practical constraints of using these cameras for the application under consideration, inexpensive 'light and camera' option instead of video recorded camera was investigated. Light and camera option utilises a series of inexpensive LEDs and cameras that can act as a virtual fence to detect the presence of elephants in between. It 
is suggested that the camera would continuously take pictures and processes the presence of light spot. Even though this option is a non-invasive and cost efficient, there are some practical limitations due to the characteristics of the application/site. Maintaining the system reliability in daytime due to daylight and in night time due to moonlight is a challenge. Also, proper installation of the virtual fence with exact alignment of lights is impractical due to the characteristics of the venue.

\subsection{Ultrasound Option}

Ultrasound option is generally used to locate animals who have the ability to detect ultrasound [12] such as bats[13], dolphins, dogs, and cats. This technique is also known and termed as sonography, ultrasonography, and Doppler study. The technique is a non-invasive diagnostic medical technique that passes ultrasound through the body of animals that produces echoes, which can identify distance, size and shape of them. A research study[14] uses Doppler principle to detect wildlife crossing the roads, in order to warn drivers. Even though the ultrasound imaging can be used to locate the elephants accurately, the main limitation is that the ultrasound does not reflect clearly from bone or air, so it needs additional rotating techniques. In addition, the operating distance is a limitation. As the sound intensity decreases rapidly with distance, the range is limited only to a few meters.

\subsection{Infra sound and Seismic Communication Option}

Infra sound option uses the ultrasound emission of elephants. Seismic option[15] uses ground pressure due to elephant movements to detect them and proximity techniques to locate them. Elephant infrasonic vocalisations are used as a means to detect[16, 17] and to determine the size and composition of the herd, the sexual state, as well as the emotional condition of an elephant[18]. These approaches have the added advantage of extending the approach to a communication strategy for chasing the elephants back to the jungle because elephant possesses the unusual ability to detect infra sound, and also they respond to distant playbacks of lowfrequency con specific calls [19,20] and long-distance, low-frequency communication[21]. However, there are significant technological challenges in hardware and technological complexities in infra sound and seismic communication devices. The buried sensors will have power problems as well. Moreover, this technique requires domain expertise in elephant communication to continue further investigations.

\subsection{Wireless Sensor Option}

Majority of the wireless or sensor network based animal monitoring/detection techniques are invasive where the animals are being tagged [22-24] or a tracking device is used [25]. These techniques are impractical for the application under consideration, because of the large extent of area to be covered and the large potential elephant population which would need tagging. The non-invasive Radio Frequency (RF) techniques are investigated as an alternative for the eleAlert. Variations in received signal strength are used to find intrusion activity at home by defining an acceptable range for the variations [26]. RF fingerprinting is widely used in localization and fingerprinting $[27,28]$ without using additional sensor components. RSS fading measurements on the links are used to motion density estimation in device free localisation in wireless sensor networks [29] with various noise reduction techniques [30]. The device free localisation is used in residential monitoring [31]. A research study [32] uses a similar approach with wireless sensor network, to measure and analyze the attenuation in wireless links to detect vegetation. However, multi-path propagation poses a serious limitation for robustness of the technique. Thus, for a robust and reliable system, removal of multi-path effects as well as thorough on site testing is 
needed. Also, best configuration and positioning of transmitters and detectors need to be determined. Energy efficiency of the sensors has to be taken in to account in real implementation.

\section{A WIRELESS SENSOR NeTWORK BASEd INTRUSION AlERT SySTEM (WI- ALERT)}

The wireless solution is selected as the best practical alternative based on the study of possible alternative technologies and applicability of each solution to the given problem domain. The concept of RF fingerprinting is extended to detect intruding elephants through identifying abnormal conditions. Due to the technical challenges of the approach, the project was divided in to three phases. The first phase was initial feasibility of the approach which is composed of prototype sensor mote development and experiments to test the signal propagation, attenuation and multi-path effects. These experiments were conducted at the University of Moratuwa in the vicinity of a small road lying through thin vegetation (Site 1). The second phase of the project is composed of network testing with the intruders/elephants at the National Zoological Gardens, Colombo (Site 2). The third phase of the project is real on site implementation and testing of the whole system. This article focuses on the first stage of initial feasibility study and the prototype experiments conducted with the elephants.

\subsection{Sensor Network}

In-house developed RF module(s) and one $433 \mathrm{MHz}$ MICA2 mote from Cross-Bow Technologies were used in the experiments. The sensor nodes developed in-house are based on the Arduino UNO board, an Xbee PRO series II module. The frequency of communication used is $2.4 \mathrm{GHz}$. Figure 2 illustrates the sensor mote we developed.

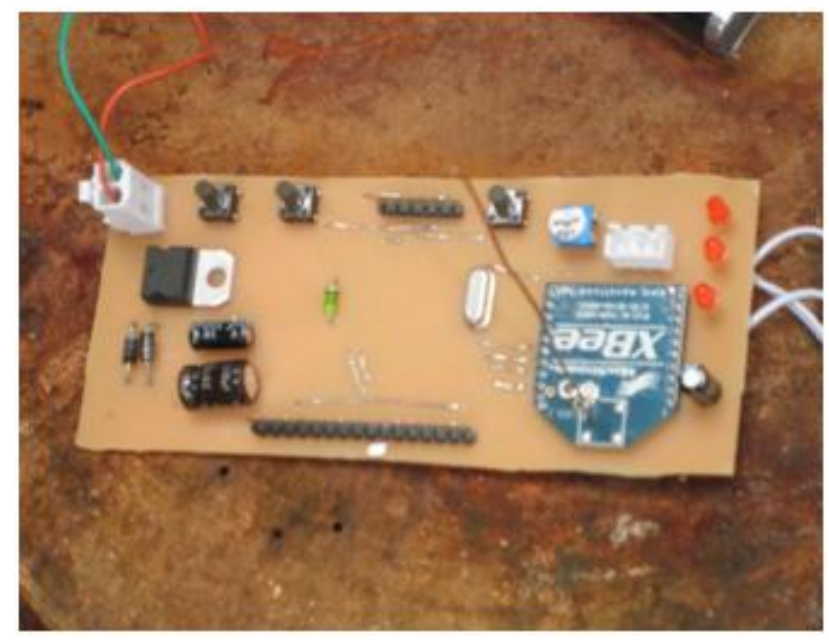

Figure 2. Xbee PRO series II sensor Mote

We used outdoor Receiver Signal Strength Indicator (RSSI) measurements in a mesh network of sensors as depicted in Figure 3. We processed the measured RSSI values in order to remove mutli-path propagation effects while detecting the presence of the intruder.

\subsection{RSSI Signal Processing of Multi-path Propagation Effect}


Most of the finger printing techniques are hindered by the problem of multi-path propagation. Even though the transmitter and the receiver are kept fixed, in a multi-path environment, the received signal is subjected to frequent fluctuations due to multi-path effect of various movements from sources such as vehicles, people and vegetation.

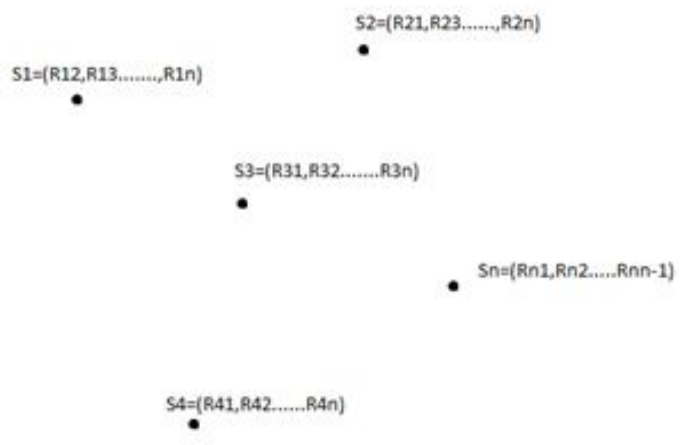

Figure 3. Intrusion Sensing Network

The most commonly used technique for removing RSSI fluctuations due to multi-path is simple time averaging. A novel approach for extracting a robust signal feature from RSSI measurements in indoor wireless LAN environments is investigated in [27]. The dynamic multi-path behaviour, which can be modelled by a convolution operation in the time domain, is transformed into an additive random variable in the logarithmic spectrum domain. Thus, the convolution process becomes a linear and separable operation which can then be effectively removed [27]. We investigated the application of this technique to outdoor environments in this study.

Figure 3 illustrates the sensor network which is composed of M sensor motes. Each sensor keeps a fingerprint of the environment, which is a vector consisting of RSSI measurements from each of the other sensors within the hearing range. The RSSI vector of the $\mathrm{i}^{\text {th }}$ sensor node is expressed in Equation (1).

$$
S_{i}=\left[r_{i, 1} r_{i, 2} \ldots \ldots r_{i,(i-1)}, r_{i,(i+1)} \ldots \ldots r_{i, M}\right]
$$

where $r_{i, j}$ is the RSSI at node $i$ from node $j$.

Let $\mathrm{y}^{\mathrm{m}}(n)$ represent the measured RSSI at time instant $n$ from the $m^{\text {th }}$ node, $x^{\mathrm{m}}(n)$ represents the decayed LOS signal in free space, $v^{\mathrm{m}}(n)$ represents the communication noise. The time varying multi-path is captured by $h^{\mathrm{m}}(n)$ representing the channel attenuation for each delayed signal.

Let $L$ be the number of delayed paths so that the measured signal from the $m^{\text {th }}$ node is given by,

$$
\mathrm{y}^{m}(n)=\sum_{l=0}^{L} h^{m}(l)\left[x^{m}(n-l)+v^{m}(n-l)\right]
$$

where, $1<m<M, 0<l<L-1,0<n<N-1$

$M$ is the number of nodes and $N$ is the number of RSSI measurement samples observed.

$$
y^{m}(n)=x^{m}(n) \otimes h^{m}(n)+v^{m}(n) \otimes h^{m}(n)
$$


Where $\otimes$ denotes the convolution operation. A model for such a channel is shown in Figure 4 .

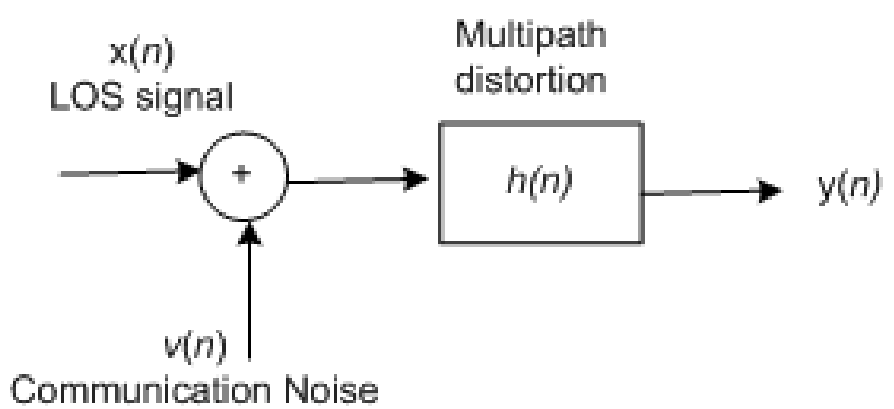

Figure 4. Model for RSSI variation in a multi-path[27]

Mitigating RSSI fluctuations due to multi-path effects is done by transforming the RSSI in to the $\log$ frequency domain using the following steps shown in Equation (4).

$$
R_{y}^{m}(k)=\frac{1}{(N-k)} \sum_{m=0}^{N-1} \mathrm{y}^{m}(n) \cdot \mathrm{y}^{m}(n+k)
$$

Where $0<k<K-1$ and $k$ is the index of autocorrelation function and $K$ is the length of the processing window.

Using above definition it can be derived that:

$$
R_{y}^{m}(k)=R_{x}^{m}(k) \otimes h^{m}(k) \otimes h^{m}(-k)+R_{v}^{m}(k) \otimes h^{m}(k) \otimes h^{m}(-k)
$$

$R_{y}^{m}(k), R_{x}^{m}(k)$ and $R_{v}^{m}(k)$ represent autocorrelation sequences for measured RSSI, LOS signal and communication noise. Taking the discrete Fourier Transform,

$$
S_{y}^{m}(f)=\left[S_{x}^{m}(f)+S_{v}^{m}(f)\right] \cdot\left|H^{m}(f)\right|^{2}
$$

$S_{y}^{m}(f), S_{x}^{m}(f)$ and $S_{v}^{m}(f)$ represent the power spectra of measured RSSI, LOS signal and communication noise respectively. The frequency index is $f$ with $F$ being the length of the power spectrum. Here $0<f<F-1$ and $1<m<M, M$ is the total number of nodes in the mesh.

Taking the logarithm of both sides of Equation (6) the log-spectrum of $y^{m}$ (n)is derived as,

$$
\log S_{y}^{m}(f)=\log \left[S_{x}^{m}(f)+S_{v}^{m}(f)\right]+2 \log \left|H^{m}(f)\right|
$$

The multi-path component has been converted to an additive random variable, whose effect can be removed by averaging. Thus, the quantity LSA_RSS ${ }^{\mathrm{m}}$ for a given point is defined as,

$$
\text { LSA_RSS }^{m}=\frac{1}{F} \cdot \Sigma_{f=0}^{T} \log S_{y}^{m}(f)
$$


The LSA_RSS ${ }^{m}$ in Equation (8) composes of the robust feature extracted from RSSI measurements by eliminating the variations due to multi-path.

For accuracy comparison purposes, the time-averaged RSSI measurement from the $m^{\text {th }}$ node is expressed as:

$$
T A \_R S S^{m}-\frac{1}{N} \cdot \Sigma_{n=0}^{N-1} y^{m}(n)
$$

\section{EXPERIMENTS AND RESULTS}

\subsection{Experiment 1: Robustness of LSA_RSS to reduce multi-path effect}

In experiment 1, measurements were used to test the robustness of LSA_RSS in order to reduce the multi-path effects at the University of Moratuwa site (Site 1).

The LSA_RSS was calculated using Equation (8) by segmenting the measurement data into nonoverlapping windows (Segmented LSA_RSS) as well as using a moving window (Moving LSA_RSS). The time averaging computation in Equation (9) was done for a simple moving window (Moving TA_RSS) for the comparison.

Figure 5 shows the results for a set of measurements taken. Sharp fluctuations in RSSI were noted to be LOS obstructions caused by the movement of people and vehicles. The smaller-scale fluctuations are due to multi-path propagation.
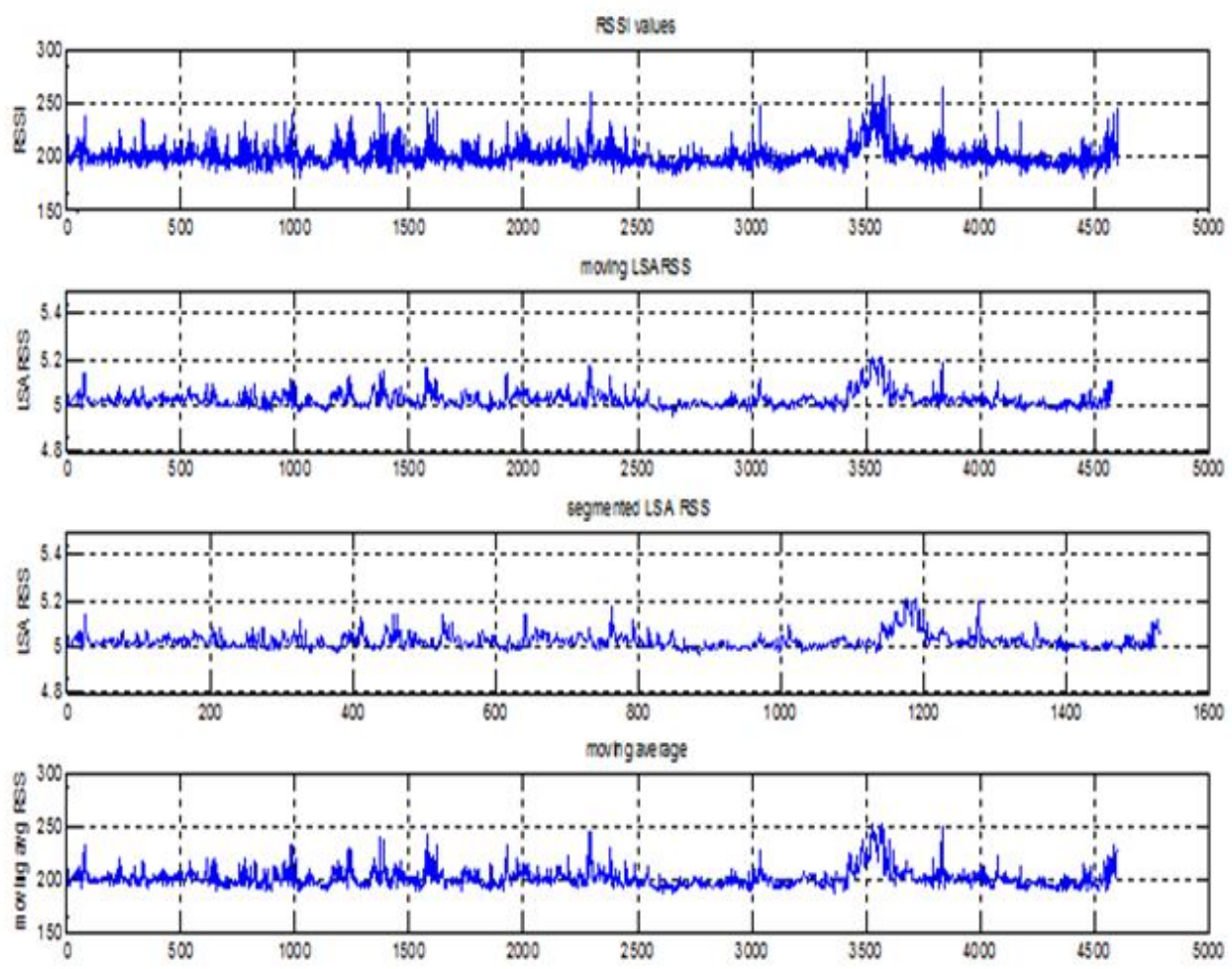

Figure 5. RSSI measurements and LSA_RSS and TA_RSS results with window size of 3 (Measurements at Site 1) 
Qualitative evaluation of the Model for RSSI variation in a multi-path channel (Figure 5) shows that the fluctuations are reduced considerably in the LSA_RSS technique compared to TA_RSS. It is also seen that in LSA_RSS, fluctuations due to obstructions are retained while those due to multi-path propagation are smoothed.

Table 2 summarises the statistical parameters of the data presented in Figure 5. These results show the significant quantitative improvement in robustness obtained with the LSA_RSS processing compared to the raw RSS measurements and the TA_RSS.

Table 2. Statistical characteristics of the data set in Figure 5.

\begin{tabular}{ccccc}
\hline & RSSI & $\begin{array}{c}\text { Moving } \\
\text { TA_RSS }\end{array}$ & $\begin{array}{c}\text { Moving } \\
\text { LSA_RS } \\
\mathbf{S}\end{array}$ & $\begin{array}{c}\text { Segmented } \\
\text { LSA_RSS }\end{array}$ \\
\hline Mean $(\boldsymbol{\mu})$ & 201.2015 & 201.1883 & 5.0235 & 5.0236 \\
\hline Std. Dev. $(\boldsymbol{\sigma})$ & 9.7662 & 8.3568 & 0.0325 & 0.0329 \\
\hline $\boldsymbol{\sigma} / \boldsymbol{\mu}$ & 0.04854 & 0.04154 & 0.00647 & 0.00655 \\
\hline
\end{tabular}

\subsection{Experiment 2: Ability to Detect Elephants}

In the experiment 2, the measurements were taken with the presence of the elephants to test the ability to detect them. The site at the Colombo National Zoological Gardens (Site 2) is on the route where elephants were taken for bathing. This is a thicker green area, surrounded by trees where significant multi-path propagation is expected. Figure 6 illustrates the site. RSSI measurements were taken with and without elephants and/or other obstructions. 

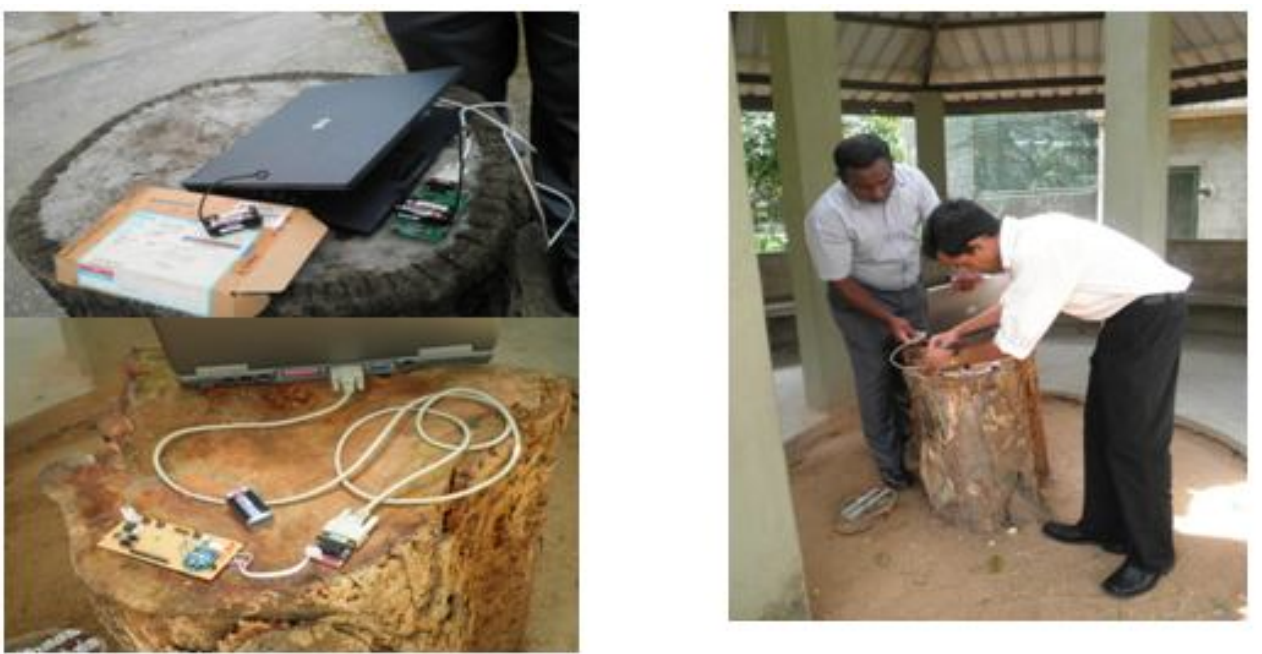

(a) Configuring the experimental network

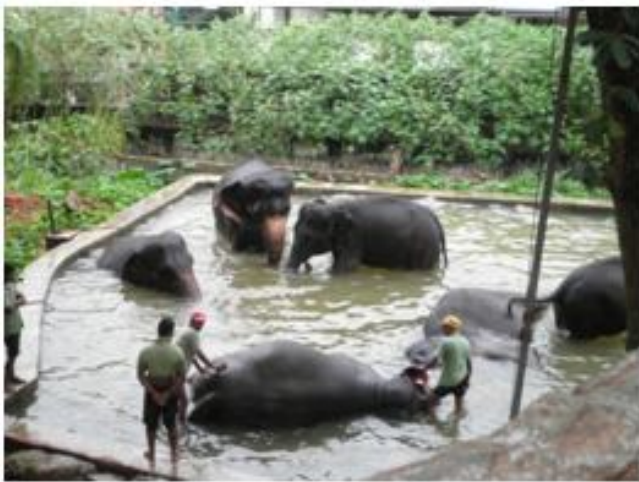

(b) Elephants used in the experiments

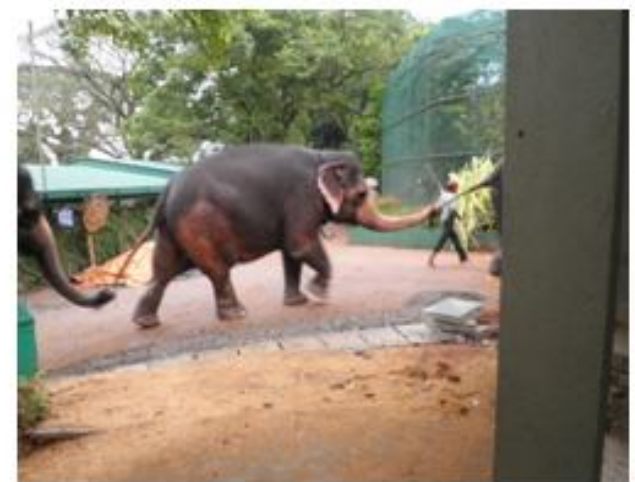

(c) Elephants' presence

Figure 6. Experimental Site in the Colombo Zoo (Site 2)

In this experiment we studied the possibility of using LSA_RSS for detecting elephants through detection of LOS obstructions, and compared the results with the same for TA_RSS.

The Probability Density Functions (PDF) and the histograms of the processed data were used as tools to identify LOS obstructions. Data collected from experiment 2 with and without LOS obstructions were analysed. The LOS obstructions were caused by elephants being present between the transmit node and the receive node(s). The processing was done using Moving TA_RSS and Moving LSA_RSS with window sizes of 3, 4 and 5.

Since the amount of data collected with LOS obstructions was relatively small, we compared the PDF of the data under normal conditions (no LOS obstructions) with the histogram of the data with obstructions. The same bin size is taken for the computation of the PDF and the histogram. We evaluated the extent to which the histogram falls outside the PDF as a quantitative measure of the technique's ability to identify obstructions.

Figure 7 shows the PDF and histograms obtained for measurements of experiment 2. Observation of Figure 7 indicates qualitatively, how the LSA_RSS technique is able to identify RSSI variations due to LOS obstructions from those due to multi-path Figure 7(b), while these are not clearly differentiated in pure RSSI measurements Figure 7(a) or with TA_RSSI Figure 7(c). 

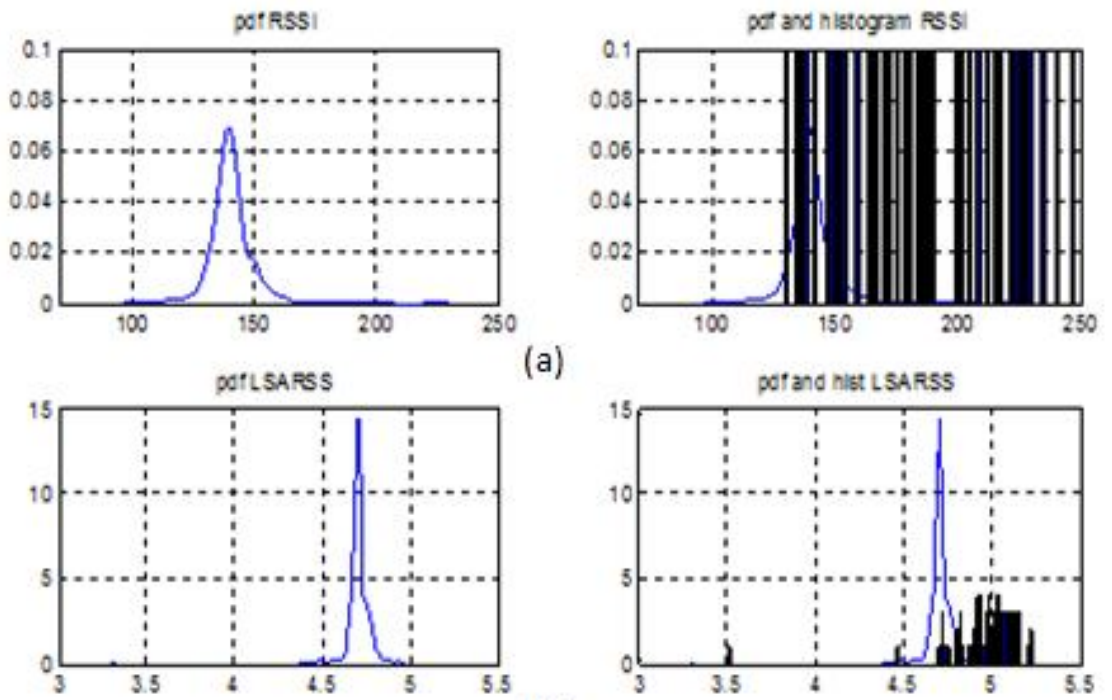

(a)
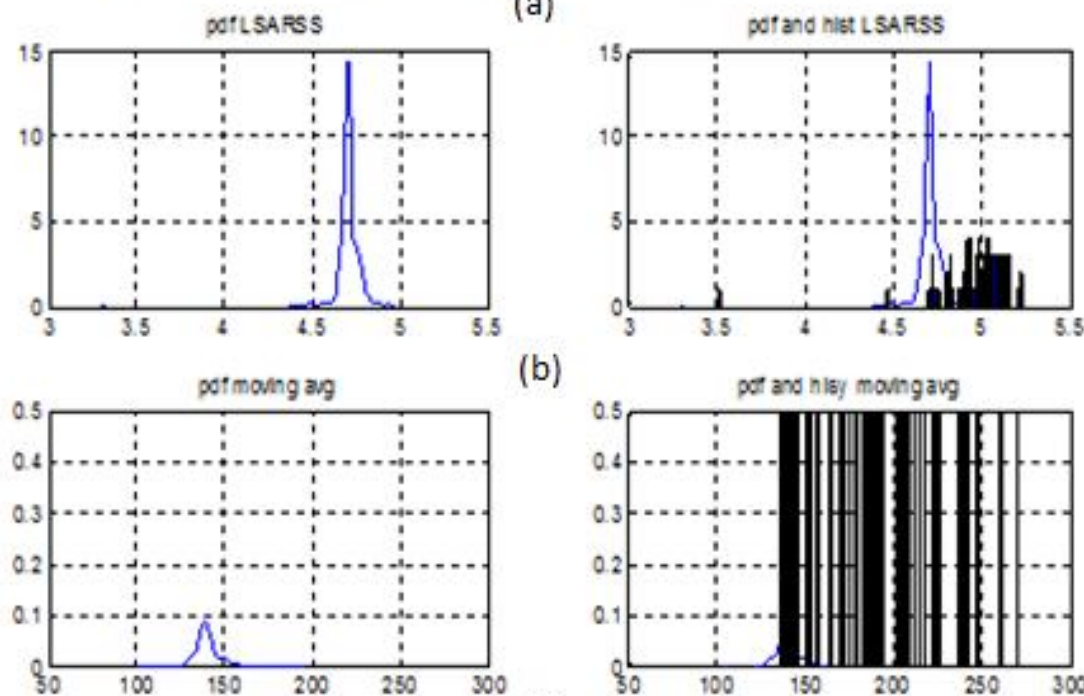

(b)

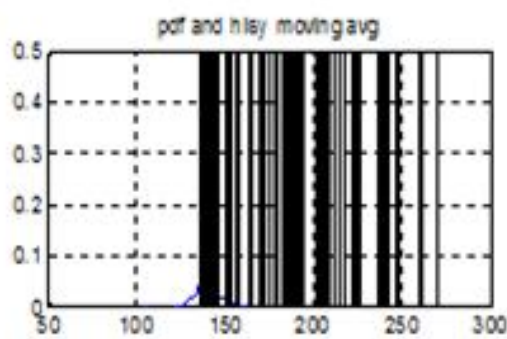

(c)

Figure 7. PDF (without LOS obstructions) and histogram (with LOS obstructions) of RSSI, Moving LSA_RSS and Moving TA_RSS for measurements taken with window size of 3. (Measurements taken at Site 2)

The measure which was defined to quantify the above observations is expressed as:

$$
\frac{\sum_{i=t}^{R} f_{i}}{\sum_{i=1}^{R} f_{1}}
$$

Where $t$ is the index of the histogram which corresponds to the value for which the PDF integrates to 1 , and $R$ is the total number of bins associated with the histogram. Thus, $P$ evaluates the fraction of measurements which fall outside the PDF of RSSI under normal circumstances.

The P-values calculated as per Equation (10) are summarised in Table 3. These results show that the LSA_RSS is superior to TA_RSS in detecting LOS obstructions. The P-value shows an increase of $36.6 \%$ for the LSA_RSS technique compared to the TA_RSS technique.

Table 3. P values for measurements depicted in Figure 7

\begin{tabular}{cccc}
\hline $\begin{array}{l}\text { Window } \\
\text { Size }\end{array}$ & RSSI & $\begin{array}{l}\text { Moving } \\
\text { TA_RSS }\end{array}$ & $\begin{array}{l}\text { Moving } \\
\text { LSA_RSS }\end{array}$ \\
\hline 3 & 0.1311 & 0.4915 & 0.5424 \\
\hline 4 & 0.1311 & 0.5172 & 0.7069 \\
\hline 5 & 0.1311 & 0.5789 & 0.7193 \\
\hline
\end{tabular}


From the results presented, we conclude that RSSI measurements from sensor nodes installed in threatened environments can be used effectively to detect the presence of elephants.

\section{Conclusions}

The need for an alternative solution arose due to the practical limitations of the existing elephant alert system. Sensor network based solution is chosen as the best alternative among many others based on various practical implications and constraints. This article reports the results of the initial phases of an ongoing research project called Wi-Alert which is a wireless sensor network based elephant detecting and alerting system.

The first phase experiments of the prototype verified the feasibility of reducing multi-path effect and the ability to filter the presence of obstacles. The second phase experiments conducted in the presence of elephants proved the ability of the prototype to detect elephants. Hence, the two stage experiments lead to the third stage of system development.

The project is ongoing and future work includes the pilot system development and real on site implementation.

\section{ACKNOWLEDGEMENTS}

Authors acknowledge the funding and support provided by Endeavour postdoctoral award by Department of Education, Employment and Workplace Relations (DEEWR), Australia to conduct the research. Also, authors wish to thank Sri Lanka Wildlife Conservation Society for providing the background for the research and the director and the medical staff of the National Zoological Gardens in Sri Lanka for providing access to the sites and facilitating experiments reported in this work.

\section{REFERENCES}

[1] R. F. W. Barnes, "The conflict between humans and elephants in the central African forests," Mammal Review, vol. 26, pp. 67-80, 1996.

[2] R. Bhima, "Elephant status and conflict with humans on the western bank of Liwonde National Park, Malawi.," Pachyderm, vol. 25, pp. 74-80, 1998.

[3] W. Kiiru, "The current status of elephant-human conflict in Kenya," Pachyderm, vol. 19, pp. 15-19, 1995.

[4] N. Ngure, "People-elephant conflict management in Tsavo, Kenya. ," Pachyderm, vol. 19, pp. 20-25, 1995.

[5] R. Sukumar, Ed., Elephant-Man conflict in Karnataka. (Karnataka-State of environment report 198485. . Bangalore, India: Centre for Taxonomic Taxonomic Studies, 1986, p.^pp. Pages.

[6] L. Naughton, et al., "The social dimensions of human-elephant conflict in Africa: A literature review and case studies from Uganda and Cameroon. ," Human-Elephant Task Conflict Task Force, of IUCN, , Glands, Switzerland.1999.

[7] S. S. Bist, "An Overview of Elephant Conservation in India," The Indian Forester, vol. 128, p. 127. 2002.

[8] B. C. Nigam, "Elephants of Jharkhand - Increasing Conflicts with Man," The Indian Forester, vol. 128, pp. 189-196, 2002.

[9] M. Crawley. (2001). Kenya mounts a game plan to cut elephant counts. Available: http://www.csmonitor.com/2001/0912/p7s2-woaf.html,

[10] L. Wijesinghe, et al., "Electric Fence Intrusion Alert System (eleAlert)," in IEEE Global Humanitarian Technology Conference, Seattle, WA, USA . 2011, pp. 46 - 50.

[11] R. Kays, et al., "Monitoring wild animal communities with arrays of motion sensitive camera traps," 2010. 
International Journal of Distributed and Parallel Systems (IJDPS) Vol.4, No.4, July 2013

[12] S. Anitei. (2007, 02 December). Top 7 Ultrasound emitting animals. Available: http://news.softpedia.com/news/Top-7-Ultrasound-Emitting-Animals-74541.shtml

[13] I. BCM. (2009). Bat Detector Hardware. Available: http://www.batmanagement.com/Ordering/acoustic/hardware.html

[14] F. Viani, et al., "WSN-based early alert system for preventing wildlife-vehicle collisions in Alps regions. ," presented at the IEEE-APS Topical Conference on Antennas and Propagation in Wireless Communications (APWC), Torino. , 2011.

[15] K. E. O'Connell-Rodwell, "Keeping an "Ear" to the Ground: Seismic Communication in Elephants " Physiology, vol. 22, pp. 287-294., 2007.

[16] G. Doluweera, et al., " Infrasound - Can it be more than a means of communication between elephants?," presented at the Symposium on Human Elephant Relationships and Conflicts, Colombo, Sri Lanka, 2003.

[17] J. V. Wijayakulasooriya, "Automatic recognition of elephant infrasound calls using formant analysis and Hidden Markov Model. ," presented at the 6th IEEE International Conference on Industrial and Information Systems (ICIIS), Kandy, Sri Lanka., 2011.

[18] P. J. Venter and J. J. Hanekom, "Automatic detection of African elephant (Loxodontaafricana) infrasonic vocalisations from recordings. ," Biosystems engineering, vol. 106, pp. 286-294., 2010.

[19] J. W. R. Langbauer, et al., "African Elephants Respond to Distant Playbacks of Low-Frequency Conspecific Calls. ," The journal of experimental biology., 1990.

[20] K. McComba, et al., "Long-distance communication of acoustic cues to social identity in African elephants.," Animal Behaviour, vol. 65, pp. 317-329, 2003.

[21] M. Garstang, "Long-distance, low-frequency elephant communication," J Comp Physiol A NeuroetholSens Neural Behav Physiol, vol. 190, pp. 791-805., 2004.

[22] V. Dyo, et al., "Evolution and sustainability of a wildlife monitoring sensor network " in the 8th ACM Conference on Embedded Networked Sensor Systems, 2010, pp. 127-140.

[23] P. Juang, et al., "Energy-efficient computing for wildlife tracking: design tradeoffs and early experiences with ZebraNet.," San Jose, CA.2002.

[24] J. Karlsson, et al., "Tracking and Identification of Animals for a Digital Zoo. ," in IEEE/ACM International Conference on Green Computing and Communications \& 2010 IEEE/ACM International Conferenceon Cyber, Physical and Social Computing, 2010, pp. 510-515.

[25] R. Ranjan and H. Shi, "'GLOBETRACKER"-A Wireless Tracking System," International Journal of Computer Networks \& Communications (IJCNC), vol. 1, pp. 125-130, 2009.

[26] F. Kausar, et al., "Intelligent Home Monitoring Using RSSI in Wireless Sensor Networks," International Journal of Computer Networks \& Communications (IJCNC) vol. 4, pp. 33-46, 2012.

[27] S. Fang, et al., "A Novel Algorithm for Multipath Fingerprinting in Indoor WLAN Environments," IEEE Transactions On Wireless Communications, vol. 7, 2008.

[28] T. S. Rappaport, et al., "Position location using wireless communications on highways of the future," IEEE Commun .Mag, vol. 34, pp. 33-41., 1996.

[29] N. Patwari and J. Wilson, "RF Sensor Networks for Device-Free Localization and Tracking," IEEE, vol. 98, pp. 1961-1973, 2010.

[30] Y. Zhao and N. Patwari, "Noise Reduction for Variance-Based Device-Free Localization and Tracking," in Eighth IEEE Communications Society Conference on Sensor, Mesh and Ad Hoc Communications and Networks (SECON), 2011, pp. 179-187.

[31] O. Kaltiokallio, et al., "Follow @ grandma: long-term device-free localization for residential monitoring," in 7th IEEE International Workshop on Practical Issues in Building Sensor Network Applications (SenseApp 2012), 2012.

[32] P. Mestre, et al., "Vegetation Growth Detection UsingWireless Sensor Networks. ," in the World Congress on Engineering.WCE 2010, London, U.K, 2010. 